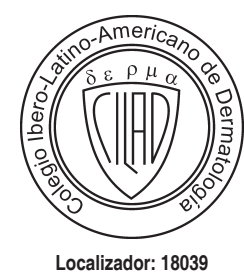

doi: $10.35366 / 91761$

\title{
Cilindroma solitario
}

\author{
Exophytic neoformation on scalp
}

Diana Elizabeth Medina Castillo,* Jorge Manuel Carbajal Méndez,

Débora Salero Martínez, ${ }^{\S}$ José Ángel Martínez Muñoz

Medicina Cutánea

Ibero-Latino-Americana

https://dx.doi.org/10.35366/91761

Palabras clave: Cilindroma, cilindroma solitario, tumor de anexos.

Keywords: Cylindroma, solitary cylindroma, adnexal tumor.

* Dermatólogo, Consulta Privada Médica Comonfort Metepec, Estado de México. "Médico Pasante, Servicio Social Facultad de Medicina Universidad Anáhuac Norte Huixquilucan, Estado de México.

${ }^{\S}$ Residente de segundo año de Medicina Interna, en el Hospital Metropolitano Grupo Ángeles.

" Dermatopatólogo, Consult Privada World Trade Center Ciudad de México.

Conflicto de intereses: Ninguno.

Recibido:

03/Junio/2018

Aceptado:

11/Junio/2019

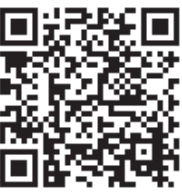

\section{REsUMEN}

El cilindroma solitario es un tumor benigno de anexos, que puede ser de origen ecrino o apocrino y su etiología es hasta el momento desconocida. Se les clasifica en dos grupos: los de carácter hereditario, que evolucionan con el tiempo a una gran masa tumoral llamada «tumor en turbante», y los esporádicos, solitarios. Es un tumor más frecuente en mujeres que en hombres. La histopatología es el estudio obligado para emitir un diagnóstico de certeza. Comunicamos un caso de cilindroma solitario y se realiza una breve revisión del tema.

\section{ABstract}

The solitary cylindroma is a benign tumor of annexes, of eccrine or apocrine origin, its origin is still under study. They are classified into two groups: those of hereditary character, which evolve over time to a large tumor mass called a turban tumor, and sporadic solitary. It is a neoplasm twice more common in women than in men. Histopathology is the study required to issue a diagnosis of certainty. We report a solitary cylindroma case and a brief review of the subject is made.

\section{INTRODUCCIÓN}

E I cilindroma cutáneo es un tumor poco frecuente que se origina en la piel cabelluda y estructuras anexiales de la cara. El término cilindroma surgió por su forma cilíndrica vista en sección transversal, y fue usado por primera vez en 1856 por Billroth para describir un tumor de orbita con apariencia hialina. ${ }^{1-3}$

En la actualidad, se reconocen tres formas de cilindromas:

1. El cilindroma cutáneo benigno, que puede ser aislado o en múltiples lesiones.

2. El cilindroma salivar maligno, también Ilamado carcinoma adenoide quístico.

3. El cilindroma maligno, el cual podemos encontrar con mayor frecuencia en el síndrome de Brooke-Spiegler. ${ }^{2,4}$

La histopatología es el estudio de elección para emitir un diagnóstico definitivo.

\section{CASO CLínICO}

Mujer de 35 años de edad, sin antecedentes patológicos de importancia, quien acude a consulta por presentar una dermatosis de tres años de evolución, localizada en la piel cabelluda, en la región parietal derecha, unilateral y asimétrica, constituida por una neoformación exofítica, hemiesférica superficie lisa, se observan telangiectasias en la superficie, puntilleo negruzco, consistencia semiblanda y renitente; asintomática (Figura 1). Se realizó excisión completa de la lesión y se envía a estudio histopatológico, con diagnóstico clínico de quiste triquilémico vs tumor de anexos.

La histopatología demostró proliferación de células basaloides, formando islotes de bordes bien definidos que se encuentran rodeados por una membrana eosinófila PAS positiva, dando un aspecto de rompecabezas (Figuras 2 y 3 ). De acuerdo a lo anterior, se emite el diagnóstico de cilindroma. El tratamiento de la lesión fue la excisión completa, se revisó al paciente durante tres años sin recidiva de la lesión.

\section{DISCUSIÓN}

El presente caso corresponde a un Cilindroma Solitario. Es una neoformación benigna dos veces más frecuente en mujeres que en hombres, estas lesiones se presentan con mayor frecuencia en la pubertad o inicio de la edad adulta. ${ }^{3,5}$ El cilíndroma solitario característica- 
mente se encuentra en el cuero cabelludo y generalmente ausencia de historia familiar. El tipo múltiple muestra pápulas y nódulos que pueden llegar a cubrir la totalidad de la piel cabelluda, dando una apariencia de turbante. Se heredan generalmente de forma autosómica dominante y puede estar asociada a otros tipos de tumores cutáneos. ${ }^{1,6}$ Existen controversias sobre si su origen se encuentra en las glándulas ecrinas o apocrinas o incluso en el epitelio folicular, ya que el tumor aparece exclusivamente en áreas vellosas ausente en la región palmoplantar. ${ }^{1}$

La mayoría de las veces se presentan como lesiones de aspecto nodular, alopécicos, firmes, lisos, rosados, de crecimiento lento, que miden entre 0.5 a $0.6 \mathrm{~cm}$ principalmente en piel cabelluda. ${ }^{7}$ Las formas solitarias, al tener una presentación clínica poco característica, hay que diferenciarlas clínicamente del carcinoma basocelular, quiste epidérmico o espiradenoma, ya que estos dos los podemos localizar en piel cabelluda y pueden mostrar aspecto quístico o pseudoquístico. ${ }^{8-10}$ Los cilindromas son en general benignos, pero pueden volverse malignos en algunas ocasiones. ${ }^{1}$

Cuando estos tumores aparecen en el contexto de la cilindromatosis múltiple, se puede presentar el síndrome de Brooke-Spiegler, donde las lesiones presentan crecimiento rápido desde el inicio, con ulceración, dolor y crecimiento local que excede los $20 \mathrm{~cm}$ e incluso la posibilidad de metástasis. ${ }^{1}$

La histopatología confirma el diagnóstico, en la cual se observa una neoformación dérmica, los cilíndromas demuestran islotes irregulares que comprenden células basaloides rodeadas por una cubierta hialina eosinofílica, un material hialino PAS +, tipo membrana, con un patrón de mosaico o de rompecabeza. ${ }^{1,11,12}$ Las islas tumorales suelen presentar dos grupos de células: células periféricas

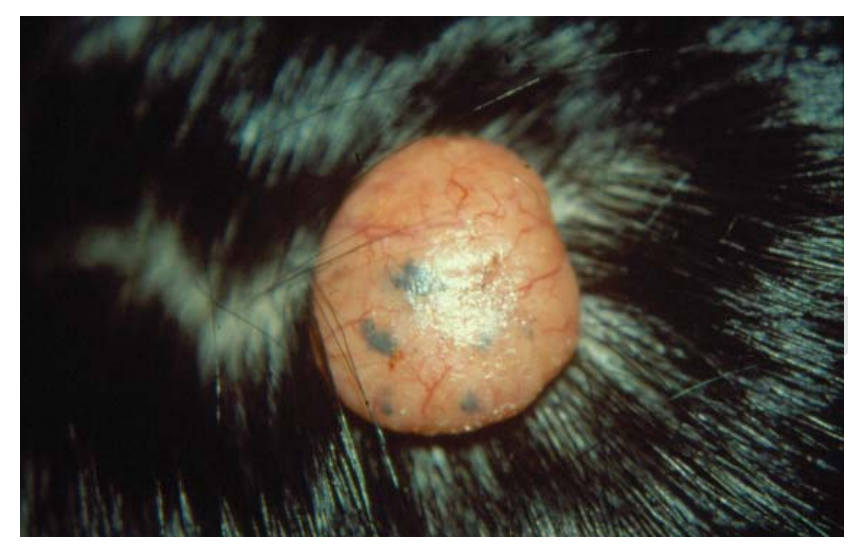

Figura 1: Aspecto clínico de la neoformación exofitica.

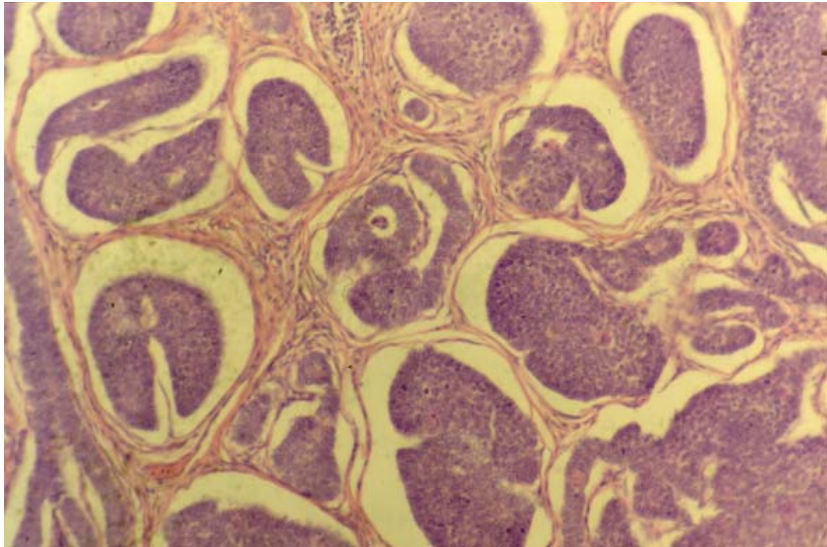

Figura 2: Microfotografía que expone la proliferación de células basaloides rodeados de membrana eosinófila con aspecto de «mosaico».

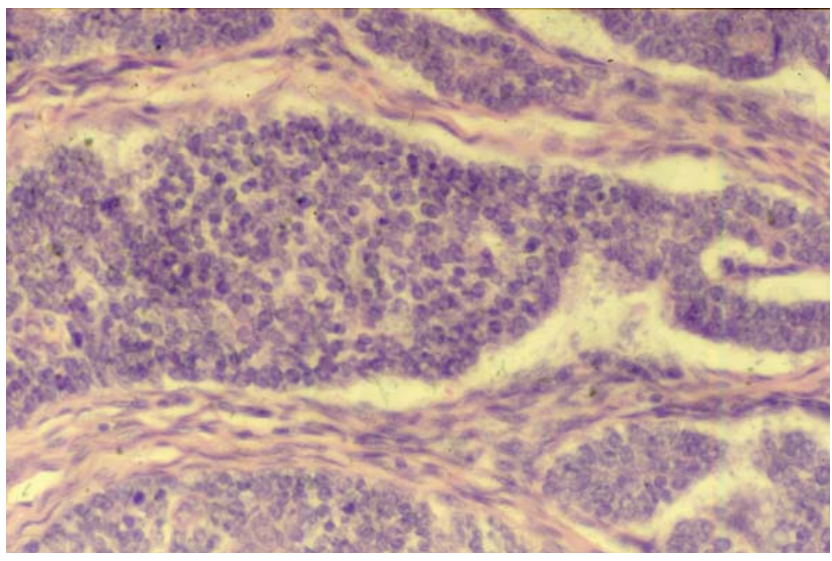

Figura 3: Microfotografía H/E 40 x, acercamiento que permite observar células periféricas con núcleos obscuros o células indiferenciadas.

con un pequeño núcleo oscuro, que representan células tumorales epiteliales indiferenciadas, y células centralizadas más diferenciadas, con un gran núcleo pálido parecido a células ductales o secretoras. ${ }^{1}$ El comportamiento agresivo se asocia con pérdida de láminas hialinas y pérdida de palidez en la periferia de las islas de células. ${ }^{1,6}$

La membrana basal de cilindromas está engrosada y presenta múltiples alteraciones, como ausencia de hemidesmosomas maduros, defectos en el procesamiento de laminina 5 y reducción de la expresión de integrina a6b4, en contraste con el aumento de la expresión de la integrina a2b1, anomalías estructurales de la membrana basal y anomalías en la expresión de las cadenas a11 y a5 de colágeno IV. ${ }^{1}$ La superregulación de la integrina ab1 
en los queratinocitos suprabasales está asociada con un aumento de la proliferación, que respalda la hipótesis de que las células madre derivadas del epitelio folicular serían las células específicas responsables de la formación del cilíndro, proporcionan argumentos por la teoría de que el origen estaría en el folículo piloso, incluso si los tumores adoptan varias vías de diferenciación, presentando formas fenotípicamente diferentes. Suponiendo que las células madre del epitelio folicular pueden ser multipolares, podrían diferenciarse en folículos, sebáceas, cepas epidérmicas y apocrinas, según el estímulo recibido. ${ }^{1}$

No es común observar la transformación maligna, debemos sospecharla ante la presencia de crecimiento rápido, ulceración, cambios de color o sangrado, principalmente en las formas múltiples. ${ }^{4}$ Los cilindromas malignos difieren de las lesiones benignas por la pérdida del patrón de tipo sierra, la pérdida de la cubierta hialina y la pérdida de la distribución celular bifásica. Hay un fuerte pleomorfismo celular con un aumento del índice mitótico. ${ }^{1}$ Es importante considerar la transformación maligna de cilindromas solitarios ya que pueden albergar potencial maligno y por lo tanto requieren una mayor vigilancia clínica. ${ }^{13}$ Síndrome de Brooke-Spiegler, cilindromas múltiples se observaron por primera vez por Brooke en 1892 y más tarde por Spiegler en 1899. También conocida como tumor de turbante o cilindromatosis familiar. ${ }^{1}$ Se clasifica por la presencia de cilindromas, tricoepiteliomas y espiradenomas en la cabeza y el cuello, generalmente a partir de la segunda década de la vida, en forma esporádica y en pacientes de mediana edad y ancianos se encuentran en trastornos hereditarios involucrados. Se presenta con crecimiento progresivo durante la vida que requiere intervenciones quirúrgicas repetidas. A pesar de que el crecimiento es lento, los síntomas son compresivos y los efectos estéticos son notables. Los nódulos pueden fusionarse en la piel cabeIluda dando como resultado un aspecto de turbante. ${ }^{1}$ Hay informes de transformación maligna de cilíndromas y la posibilidad de metástasis a los ganglios linfáticos, tiroides, hígado, pulmones y huesos. Incluso pueden infiltrarse en el cráneo, causando hemorragia y meningitis. Los pacientes con este síndrome son propensos al desarrollo de tumores anexiales múltiples, como cilindromas, tricoepiteliomas y espiradenomas. ${ }^{1}$ Los cilindromas esporádicos benignos expresan la fusión del gen MYB-NFIB. Se propone que la mutación responsable del síndrome de Brooke-Spiegler se encuentra en el gen CYLD ubicado en el cromosoma 16, con expresión variada y penetración absoluta. El gen de la cilindromatosis (CYLD) se describió como el único gen supresor responsable del síndrome. ${ }^{1}$

El reconocimiento de esta neoplasia es de importancia crítica, ya que actualmente no existe un tratamiento basado en la evidencia y la mayoría de nuestro conocimiento se basa en los resultados de algunos informes de casos y series pequeñas. ${ }^{3}$ El abordaje terapéutico dependerá del número y tamaño de las lesiones. El tratamiento de elección es la extirpación quirúrgica en los casos solitarios. ${ }^{9,10}$

En caso de tumores múltiples existen tratamientos optativos como son electrocirugía, láser con dióxido de carbono, crioterapia y radioterapia. Las indicaciones para la cirugía son ulceración, infección, sangrado y estética. La escisión quirúrgica simple y la reconstrucción con colgajos e injertos locales son suficientes para el tratamiento de cilindromas benignos. ${ }^{6}$ Los cilindromas múltiples generalmente requieren cirugía plástica extensa en procedimientos únicos o múltiples. La ventaja de la terapia quirúrgica es que logra la eliminación radical del tumor, lo que implica un riesgo mínimo de recurrencia y transformación del tumor maligno. ${ }^{14}$

Correspondencia:
Diana Elizabeth. Medina Castillo
Médica Comonfort.
Avenida Ignacio Comonfort 100,
planta baja, interior 8,
Barrio Coaxustenco Metepec,
Estado de México, CP 52140, México.
Tels: 52 (722) 2130071 y 2324265
E-mail: mecasdiderma@gmail.com

Correspondencia:

Médica Comonfort.

Avenida Ignacio Comonfort 100,

planta baja, interior 8 ,

Barrio Coaxustenco Metepec

Tels: 52 (722) 2130071 y 2324265

E-mail: mecasdiderma@gmail.com

\section{BIBLIOGRAFÍA}

1. Clarice Jordao, Taissa Canedo de Magalhães. Review cylindroma: an update. International Journal of Dermatology. 2014.

2. Cohen YK, Elpern DJ. Dermatoscopic pattern of a cylindroma. Dermatol Pract Concept. 2014; 4 (1): 67-68.

3. Bosio BM, Garay I, Ruiz LA, Kurpis M. Cilindroma solitario. Arch Argent Dermatol. 2015; 65 (1): 16-18.
4. Abedi SM, El-Shinawy I, Alowami S. Malignant cylindroma arising as a solitary neck lesion. Eur J Dermatol. 2014; 24: 403-405.

5. Jordão C, de Magalhães TC, Cuzzi T, Ramos-e-Silva M. Cylindroma: an update. Int J Dermatol. 2015; 54 (3): 275-278.

6. Hosnuter M, Babuccu O, Kargi E, Numanoglu G, Koca R, Babuccu B. Malignant solitary cylindroma of the scalp. Eur J Plast Surg. 2004; 27: 246-248. 
7. Lauro MF, Rodríguez SAÁ, S, Ciancio MR, Villa R, Peláez O. Nódulo pigmentado en rostro. Cilindroma. Arch Argent Dermatol. 2013; 63: 33-35.

8. Akgul GG, Yenidogan E, Dinc S, Pak I, Colakoglu MK, Gulcelik MA. Malign cylindroma of the scalp with multiple cervical lymph node metástasis- a case report. Int J Surg Case Reports. 2013; 4 (7): 589-592. http://doi.org/10.1016/j. ijscr.2013.02.027.

9. Akgul G, Yenidogan E, Dinc S, Colakoglu M, Gulcelik M. Malign cylindroma of the scalp with multiple cervical lymph node metastasis-A case report. Int J Surg Case Rep. 2013; 4 (7): 589-592.
10. Abedi SM, El-Shinnawy I, Alowami S. Malignant cylindroma arising as a solitary neck lesion. Eur J Dermatol. 2014; 24 (3): 403-405.

11. Cabo H, Pedrini F, Cohen SE. Dermoscopy of cylindroma. Dermatol Res Pract. 2010; 2010: 1-2.

12. Lallas A, Apalla Z, Tzellos T, Lefaki I. Dermoscopy of solitary cylindroma. Eur J Dermatol. 2011; 21 (4): 645-646.

13. Chao E. Transformación maligna de un cilindroma solitario. Journal of the American Academy of Dermatology. 2017; AB165: 5748.

14. Rončević $R$, Rončević $D$. Treatment of multiple scalp cylindroma. J Surg Dermatol. 2016; 1(2): 100-102. 